\title{
ON HYDRODYNAMIC THEORY OF QUANTUM SPIN CRYSTALS
}

\author{
A.A.ISAYEV, M.Yu.KOVALEVSKY \\ National Centre "Kharkiv Physical and Technical Institute" \\ Ukrainian National Academy of Sciences, \\ 1 Academichna St., UA-310108 Kharkiv, Ukraine
}

Received April 19, 1996

\begin{abstract}
On the base of the Hamiltonian approach, hydrodynamic equations for quantum spin crystal are obtained. Basic thermodynamic identity is formulated. Expressions for the flux densities of additive motion integrals in terms of thermodynamic potential density are found both for the cases of Galilean invariant and Galilean noninvariant systems. Stationary solutions corresponding to spiral magnetic ordering are received. Spectrum of propagating modes is studied.
\end{abstract}

\section{Introduction}

It is well known that superfluidity in a quantum crystal [1] points towards the possibility of two types of motion. The first type is the motion of lattice sites inherent for a solid, while the second type is associated with mass transfer by quasiparticles with motionless lattice sites, which is characteristic for a superfluid liquid. Linear dynamics equations for quantum crystals were obtained in Ref. [1] by using a phenomenological approach; their nonlinear generalization was carried out in Refs. [2-4]. In these works, however, the effect of spin degrees of freedom on nonequilibrium processes in quantum crystals was disregarded, although it can be significant for quantum solid ${ }^{3}$ He. Bearing in mind that the symmetry relative to uniform spin rotations is spontaneously destroyed in superfluid phases of ${ }^{3} \mathrm{He}$, we consider the case when spin invariance is broken completely (the parameter describing such breaking is the real matrix of rotation), which corresponds to the B-phase of ${ }^{3} \mathrm{He}$. In other words, we consider a quantum spin crystal whose equilibrium state is characterized by a simultaneous violation of phase, translational and spin invariance.

The variables describing violation of the symmetry as to spin rotations, phase transformations and spatial translations are the real matrix of rotation $a_{\alpha \beta}(x)$ in the spin space, the superfluid phase $\phi(x)$ and the displacement vector $u_{i}(x)$ in the configuration space respectively. Breaking of any two of these symmetries was considered earlier [1-6]. The case of simultaneous violation of all the three types of the symmetry has not yet been analysed. We will try to solve the problem formulated above by using the Hamiltonian approach. Let us briefly describe the basic concepts of this method. 


\section{Fundamentals of the Hamiltonian approach}

The Hamiltonian approach to continuous system with dynamic variables $\varphi_{\alpha}(x)$ is based on defining the Hamiltonian of the system $H \equiv \int d^{3} x \varepsilon(x)$ as a functional of dynamic variables as well as Poisson's brackets (PB) for these variables. The equation of motion of an arbitrary physical quantity $\mathrm{A}$ in the Hamiltonian method has the form:

$$
\dot{A}=\{A, H\} \text {. }
$$

PB for arbitrary functionals $A$ and $B$ of dynamic variables $\varphi_{\alpha}(x)$ are defined as

$$
\{A, B\}=\int d^{3} x d^{3} x^{\prime} \frac{\delta A}{\delta \varphi_{\alpha}(x)}\left\{\varphi_{\alpha}(x), \varphi_{\beta}\left(x^{\prime}\right)\right\} \frac{\delta B}{\delta \varphi_{\beta}\left(x^{\prime}\right)} .
$$

If the $\mathrm{PB}$ of the variables $\varphi_{\alpha}(x)$ are antisymmetric to their transposition and satisfy Jacobi's identity, the PB of arbitrary functionals $A$ and $B$ have, according to definition (1.2) similar properties:

$$
\begin{gathered}
\{A, B\}=-\{B, A\}, \quad\{A B, C\}=A\{B, C\}+B\{A, C\}, \\
\{A,\{B, C\}\}+\{B,\{C, A\}\}+\{C,\{A, B\}\}=0 .
\end{gathered}
$$

By using the Hamiltonian approach we can easily formulate the differential conservation laws associated with various symmetry properties of the Hamiltonian. The equation of motion for the density $b(x)$ of an arbitrary physical quantity $B=\int d^{3} x b(x)$ can be represented, according to Eq.(1.1), in the form

$$
\dot{b}(x)=\{b(x), H\} \equiv\{B, \varepsilon(x)\}-\frac{\partial b_{k}(x)}{\partial x_{k}},
$$

where

$$
b_{k}(x)=\int d^{3} x^{\prime} x_{k}^{\prime} \int_{0}^{1} d \lambda\left\{b\left(x+\lambda x^{\prime}\right), \varepsilon\left(x-(1-\lambda) x^{\prime}\right)\right\} .
$$

If the quantity $B$ is the generator $G$ of the symmetry group of the Hamiltonian, then Eq. (1.4) has the form of a differential conservation law. The generator $G$ in this case does not depend on time. Indeed,

$$
\dot{G}=\{G, H\}=-\{H, G\}=-\delta H,
$$

where $\delta H$ is the variation of the Hamiltonian associated with considering transformations. Therefore, $\dot{G}=0$ if $\delta H=0$.

\section{Poisson brackets and conservation laws}

It was mentioned in Introduction that in addition to the densities of entropy $\sigma(x)$, mass $\varrho(x)$, momentum $\pi_{i}(x)$ and spin $s_{\alpha}(x)$, characterizing the state of a quantum spin crystal with broken symmetry with respect to spin rotations, we must also choose the orthogonal matrix of rotation $a_{\alpha \beta}(x)$, the superfluid phase $\phi(x)$ and the displacement vector $u_{i}(x)$ as dynamic variables. Therefore the energy density $\varepsilon(x)$ in the general case is a functional of these variables

$$
\varepsilon(x)=\varepsilon\left(x ; \sigma\left(x^{\prime}\right), \varrho\left(x^{\prime}\right), \pi_{i}\left(x^{\prime}\right), s_{\alpha}\left(x^{\prime}\right), a_{\alpha \beta}\left(x^{\prime}\right), \phi\left(x^{\prime}\right), u_{i}\left(x^{\prime}\right)\right) .
$$


We assume that spin interactions in the system are of an exchange nature (we neglect weak relativistic interactions). Then the energy density $\varepsilon(x)$ is invariant as to uniform spin rotations. Considering that for rotations described by the matrix $b$, the spin $s_{\alpha}$ and the rotation matrix $a_{\alpha \beta}$ are transformed according to the formulae

$$
s \rightarrow s^{\prime}=b s, a \rightarrow a^{\prime}=a \tilde{b}
$$

we have

$$
\varepsilon\left(x ; b s\left(x^{\prime}\right), a\left(x^{\prime}\right) \tilde{b}\right)=\varepsilon\left(x ; s\left(x^{\prime}\right), a\left(x^{\prime}\right)\right),
$$

(we write the dependence only on the variables which change during transformations under investigation).

For a uniform phase transformation with a phase $\psi$ we have

$$
\phi \rightarrow \phi^{\prime}=\phi+\psi
$$

and the property of energy density invariance can be written in the form

$$
\varepsilon\left(x ; \phi\left(x^{\prime}\right)+\psi\right)=\varepsilon\left(x ; \phi\left(x^{\prime}\right)\right) .
$$

For transformation of translations by a constant vector $a_{i}$ in the configuration space we have

$$
x_{i} \rightarrow x_{i}^{\prime}=x_{i}+a_{i} .
$$

The corresponding invariance property has the form

$$
\begin{aligned}
& \varepsilon\left(x+a ; \sigma\left(x^{\prime \prime}\right), \varrho\left(x^{\prime \prime}\right), \pi\left(x^{\prime \prime}\right), s\left(x^{\prime \prime}\right), a\left(x^{\prime \prime}\right), \phi\left(x^{\prime \prime}\right), u\left(x^{\prime \prime}\right)+a\right)= \\
= & \varepsilon\left(x ; \sigma\left(x^{\prime}\right), \varrho\left(x^{\prime}\right), \pi\left(x^{\prime}\right), s\left(x^{\prime}\right), a\left(x^{\prime}\right), \phi\left(x^{\prime}\right), u\left(x^{\prime}\right)\right) ; x^{\prime \prime} \equiv x^{\prime}-a .
\end{aligned}
$$

Let us define the $\mathrm{PB}$ of dynamic variables of the system. According to Refs. [7-9] we define the complete system of PB by the equalities

$$
\begin{gathered}
\left\{\pi_{i}(x), u_{k}\left(x^{\prime}\right)\right\}=-\left(\delta_{i k}-\nabla_{i} u_{k}(x)\right) \delta\left(x-x^{\prime}\right), \\
\left\{\pi_{i}(x), \sigma\left(x^{\prime}\right)\right\}=-\sigma(x) \nabla_{i} \delta\left(x-x^{\prime}\right),\left\{\pi_{i}(x), \varrho\left(x^{\prime}\right)\right\}=-\varrho(x) \nabla_{i} \delta\left(x-x^{\prime}\right), \\
\left\{\pi_{i}(x), s_{\alpha}\left(x^{\prime}\right)\right\}=-s_{\alpha}(x) \nabla_{i} \delta\left(x-x^{\prime}\right),\left\{\pi_{i}(x), a_{\alpha \beta}\left(x^{\prime}\right)\right\}=\delta\left(x-x^{\prime}\right) \nabla_{i} a_{\alpha \beta}(x), \\
\left\{\pi_{i}(x), \pi_{k}\left(x^{\prime}\right)\right\}=\pi_{k}(x) \nabla_{i}^{\prime} \delta\left(x-x^{\prime}\right)-\pi_{i}\left(x^{\prime}\right) \nabla_{k} \delta\left(x-x^{\prime}\right), \\
\left\{\pi_{i}(x), \phi\left(x^{\prime}\right)\right\}=\delta\left(x-x^{\prime}\right) \nabla_{i} \phi(x),\left\{\varrho(x), \phi\left(x^{\prime}\right)\right\}=\delta\left(x-x^{\prime}\right), \\
\left\{s_{\alpha}(x), s_{\beta}\left(x^{\prime}\right)\right\}=\epsilon_{\alpha \beta \gamma} s_{\gamma}(x) \delta\left(x-x^{\prime}\right),\left\{s_{\alpha}(x), a_{\beta \gamma}\left(x^{\prime}\right)\right\}=\epsilon_{\alpha \gamma \rho} a_{\beta \rho}(x) \delta\left(x-x^{\prime}\right) .
\end{gathered}
$$

( $\mathrm{PB}$ of the remaining variables are equal to zero). It can be easily seen that these PB satisfy Jacobi's identity (1.3). Note that in view of the invariance of $\varepsilon(x)$ with respect to phase transformations and spatial translations the energy density $\varepsilon(x)$ depends not on the quantities $\phi(x), u_{i}(x)$ themselves, but only on their derivatives $\nabla_{i} \phi \equiv p_{i}, \nabla_{k} u_{i}$ (or, in the latter case, on the quantities $\left.b_{i k} \equiv \delta_{i k}-\nabla_{k} u_{i}\right)$. The vector $p_{i}$ has meaning of the superfluid momentum. Consequently, it is convenient to choose the quantities $p_{i}$ and $b_{i k}$ directly as dynamic variables along with other variables. For nontrivial $\mathrm{PB}$ of these variables we have

$$
\left\{\pi_{i}(x), b_{k l}\left(x^{\prime}\right)\right\}=-b_{k i}(x) \nabla_{l} \delta\left(x-x^{\prime}\right),\left\{\pi_{i}(x), p_{j}\left(x^{\prime}\right)\right\}=-p_{i}(x) \nabla_{j} \delta\left(x-x^{\prime}\right),
$$




$$
\left\{\varrho(x), p_{i}\left(x^{\prime}\right)\right\}=-\nabla_{i} \delta\left(x-x^{\prime}\right) .
$$

Using the general expression of $\varepsilon(x)$ and the PB (2.4),(2.5), we can obtain nonlocal dynamic equations for a quantum spin crystal [10].

Let us now formulate the differential conservation laws associated with the symmetry properties of the Hamiltonian of the system. For this purpose, we use the results obtained in Sec. 1. Putting in formula (1.4) $b(x)=\varrho(x)$ and supposing that the energy density satisfies the condition $\{M, \varepsilon(x)\}=$ $0, M=\int d^{3} x \varrho(x)$, we obtain the following equation

$$
\dot{\varrho}(x)=-\nabla_{k} j_{k}(x), j_{k}(x)=\int d^{3} x^{\prime} x_{k}^{\prime} \int_{0}^{1} d \lambda\left\{\varrho\left(x+\lambda x^{\prime}\right), \varepsilon\left(x-(1-\lambda) x^{\prime}\right)\right\},
$$

where $j_{k}(x)$ is the mass flux density.

If the energy density satisfies complies with the relationship $\left\{S_{\alpha}, \varepsilon(x)\right\}=$ $0, S_{\alpha}=\int d^{3} x s_{\alpha}(x)$, then for $b(x)=s_{\alpha}(x)$ we obtain the following equation $\dot{s}_{\alpha}(x)=-\nabla_{k} j_{\alpha k}(x), j_{\alpha k}(x)=\int d^{3} x^{\prime} x_{k}^{\prime} \int_{0}^{1} d \lambda\left\{s_{\alpha}\left(x+\lambda x^{\prime}\right), \varepsilon\left(x-(1-\lambda) x^{\prime}\right)\right\}$,

where $j_{\alpha k}$ is the spin flux density.

For $b(x)=\varepsilon(x)$, we get, in accordance with (1.4)

$$
\dot{\varepsilon}(x)=-\nabla_{k} q_{k}(x), q_{k}(x)=\frac{1}{2} \int d^{3} x^{\prime} x_{k}^{\prime} \int_{0}^{1} d \lambda\left\{\varepsilon\left(x+\lambda x^{\prime}\right), \varepsilon\left(x-(1-\lambda) x^{\prime}\right)\right\},
$$

where $q_{k}(x)$ is the energy flux density.

Finally, let us consider the translation transformation

$$
f(x) \rightarrow f^{\prime}(x)=f(x-y), \quad f=\left\{\sigma, \varrho, \pi_{i}, s_{\alpha}, a_{\alpha \beta}, p_{i}, b_{i k}\right\},
$$

which remaines the $\mathrm{PB}(2.4),(2.5)$ invariant. This means that there exists a functional $G$ corresponding to infinitely small translations (generator of translations) and satisfying the relations

$$
\{f(x), G\}=\delta f(x)=-\delta y_{k} \nabla_{k} f(x) .
$$

This functional has the form

$$
G=\delta y_{k} \int d^{3} x \pi_{k}(x)
$$

Let us suppose that the energy density $\varepsilon(x)$ matches the translation invariance condition:

$$
\delta \varepsilon(x)=\{\varepsilon(x), G\}=-\delta y_{k} \nabla_{k} \varepsilon(x) .
$$

Assuming that $b(x)=\pi_{k}(x)$ in relation (1.4) and taking into account (2.10), we obtain

$$
\begin{gathered}
\dot{\pi}_{i}(x)=-\nabla_{k} t_{i k}(x), \\
t_{i k}(x)=-\varepsilon(x) \delta_{i k}+\int d^{3} x^{\prime} x_{k}^{\prime} \int_{0}^{1} d \lambda\left\{\pi_{i}\left(x+\lambda x^{\prime}\right), \varepsilon\left(x-(1-\lambda) x^{\prime}\right)\right\} .
\end{gathered}
$$

Here $t_{i k}$ is the momentum flux density. 


\section{Thermodynamics. Local form of dynamic equations}

The structure of nonlocal dynamical equations [10] is simplified considerably in the longwave limit, when spatial inhomogeneities of dynamic variables are small. Assuming that the energy density is a function of the quantities $\sigma, \varrho, \pi_{i}, s_{\alpha}, p_{i}, b_{i k}, a_{\alpha \beta}, \nabla a_{\alpha \beta}$ (or, that the same, the left Cartan form $\omega_{\alpha k} \equiv$ $1 / 2 \epsilon_{\alpha \beta \gamma} a_{\lambda \gamma} \nabla_{k} a_{\lambda \beta}$ instead of $\left.\nabla a_{\alpha \beta}\right)$, i.e.,

$$
\varepsilon(x)=\varepsilon\left(\sigma(x), \varrho(x), \pi_{i}(x), p_{i}(x), b_{i k}(x), s_{\alpha}(x), a_{\alpha \beta}(x), \omega_{\alpha k}(x)\right)
$$

we obtain dynamic equations in the local form. Since the energy density satisfies the property of rotational invariance (2.3), we can write

$$
\varepsilon\left(s, \omega_{k}, a\right)=\varepsilon\left(a s, a \omega_{k}, 1\right) \equiv \varepsilon\left(\underline{s}, \underline{\omega}_{k}\right),
$$

where $\underline{s}=a s, \underline{\omega}_{k}=a \omega_{k}$ is the right Cartan form, $\underline{\omega}_{\alpha k}=1 / 2 \epsilon_{\alpha \beta \gamma} a_{\beta \lambda} \nabla_{k} a_{\gamma \lambda}$. Note that we use a formalism of left and right Cartan forms $\omega_{k}, \omega_{k}$ instead of $\nabla_{k} a, \tilde{a} \nabla_{k} a$ (that mathematically is identical) because they are the convenient compact constructions obeying the simple transformation properties relative to spin rotations. In the theory of magnetic systems with spontaneously broken spin invariance they play the role analogous to that of the superfluid momentum for a superfluid system $[9,11]$. By virtue of formulae (2.4), the following relations hold

$$
\left\{s_{\alpha}(x), \underline{\omega}_{\beta k}\left(x^{\prime}\right)\right\}=a_{\beta \alpha}\left(x^{\prime}\right) \nabla_{k}^{\prime} \delta\left(x-x^{\prime}\right),\left\{s_{\alpha}(x), \underline{s}_{\beta}\left(x^{\prime}\right)\right\}=0,
$$

leading to the invariance of the quantities $\underline{s}, \underline{\omega}_{k}$ with respect to global spin rotations:

$$
\left\{S_{\alpha}, \underline{\omega}_{\beta k}(x)\right\}=\left\{S_{\alpha}, \underline{s}_{\beta}(x)\right\}=0,
$$

We formulate the second law of thermodynamics for the system under investigation. We write the basic thermodynamic identity in the form

$$
d \varepsilon=T d \sigma+\mu d \varrho+v_{k}^{n} d \pi_{k}+\underline{h}_{\alpha} d \underline{s}_{\alpha}+\underline{\chi}_{\alpha k} d \underline{\omega}_{\alpha k}+j_{i}^{s} d p_{i}+\nu_{i k} d b_{i k} .
$$

Here $T$ is the temperature, $\mu$ is the chemical potential, $v_{k}^{n}$ is the normal velocity, $j_{i}^{s}$ is the superfluid mass current and the quantities $\underline{h}_{\alpha}, \underline{\chi}_{\alpha k}, \nu_{i k}$ are the respective conjugate variables to $\underline{s}_{\alpha}, \underline{\omega}_{\alpha k}, b_{i k}\left(h_{\alpha}=\underline{h}_{\beta} a_{\beta \alpha}\right.$ is the magnetizing field).

Let us find expressions for flux densities of mass, spin, momentum and energy corresponding to the energy density (3.2). Using formulae (2.6)-(2.8) and (2.11), we obtain

$$
\begin{gathered}
j_{k}=\varrho v_{k}^{n}+j_{k}^{s}, j_{\alpha k}=s_{\alpha} v_{n}^{k}+\chi_{\alpha k}, \\
t_{i k}=p \delta_{i k}+\pi_{i} v_{k}^{n}+p_{i} j_{k}^{s}+\omega_{\alpha i} \chi_{\alpha k}+b_{j i} \nu_{j k}, \\
q_{k}=v_{k}^{n}\left(T \sigma+\mu \varrho+v_{l}^{n} \pi_{l}+h_{\alpha} s_{\alpha}\right)+j_{k}^{s}\left(\mu+p_{i} v_{i}^{n}\right)+\chi_{\alpha k}\left(h_{\alpha}+v_{i}^{n} \omega_{\alpha i}\right)+v_{j}^{n} b_{i j} \nu_{i k},
\end{gathered}
$$

where

$$
p \equiv-\varepsilon+T \sigma+v_{l}^{n} \pi_{l}+\mu \varrho+h_{\alpha} s_{\alpha},
$$

is the pressure, $\chi_{\alpha k}=\partial \varepsilon / \partial \omega_{\alpha k}$. In accordance with the definition of energy density (3.2) it is expedient to choose along with other variables the quantities $\underline{s}, \underline{\omega}_{k}$ as independent variables too. Calculating flux densities in 
new variables by using the PB algebra $(2.4),(2.5)$, we obtain the following equations of dynamics of quantum spin crystals in the long-wave limit:

$$
\begin{gathered}
\dot{\sigma}=-\nabla_{k}\left(\sigma v_{k}^{n}\right), \dot{\varrho}=-\nabla_{k}\left(\varrho v_{k}^{n}+j_{k}^{s}\right), \\
\underline{\dot{s}}_{\alpha}=-\nabla_{k}\left(\underline{s}_{\alpha} v_{k}^{n}+\underline{\chi}_{\alpha k}\right)+\epsilon_{\alpha \beta \gamma}\left(\underline{s}_{\beta} \underline{h}_{\gamma}+\underline{\omega}_{\beta k} \underline{\chi}_{\gamma k}\right), \\
\dot{\pi}_{i}=-\nabla_{k}\left(p \delta_{i k}+\pi_{i} v_{k}^{n}+p_{i} j_{k}^{s}+\underline{\omega}_{\alpha i} \underline{\chi}_{\alpha k}+b_{j i} \nu_{j k}\right),
\end{gathered}
$$

where

$$
p=-\varepsilon+T \sigma+v_{l}^{n} \pi_{l}+\mu \varrho+\underline{h}_{\alpha} \underline{s}_{\alpha}
$$

and the equations for parameters describing the broken symmetries have the form

$$
\begin{gathered}
\dot{a}_{\alpha \beta}=-v_{i}^{n} \nabla_{i} a_{\alpha \beta}+\epsilon_{\alpha \rho \gamma} a_{\rho \beta} \underline{h}_{\gamma}, \\
\dot{p}_{i}=-\nabla_{i}\left(p_{j} v_{j}^{n}+\mu\right), \dot{b}_{i k}=-\nabla_{k}\left(b_{i j} v_{j}^{n}\right) .
\end{gathered}
$$

From Eqs. (3.5) it follows the conservation law for the energy density:

$$
\begin{gathered}
\dot{\varepsilon}=-\nabla_{k}\left[v_{k}^{n}\left(T \sigma+\mu \varrho+v_{l}^{n} \pi_{l}+\underline{h}_{\alpha} \underline{s}_{\alpha}\right)+j_{k}^{s}\left(\mu+p_{i} v_{i}^{n}\right)+\right. \\
\left.+\underline{\chi}_{\alpha k}\left(\underline{h}_{\alpha}+\underline{\omega}_{\alpha i} v_{i}^{n}\right)+b_{i j} \nu_{i k} v_{j}^{n}\right],
\end{gathered}
$$

and from the first of Eqs. (3.6) it follows the dynamic equation for the right Cartan form $\underline{\omega}_{\alpha k}$ :

$$
\underline{\dot{\omega}}_{\alpha k}=-\nabla_{k}\left(\underline{h}_{\alpha}+\underline{\omega}_{\alpha k} v_{k}^{n}\right)+\epsilon_{\alpha \beta \gamma} \underline{\omega}_{\beta k}\left(\underline{h}_{\gamma}+\underline{\omega}_{\gamma k} v_{k}^{n}\right) .
$$

The complete system of equations (3.5)-(3.7) defines the dynamic properties of a quantum spin crystal in neglect of dissipative processes. If the variable $a_{\alpha \beta}$ is cyclic (the Hamiltonian $H$ is independent of $a_{\alpha \beta}$ ), the spin dynamics of the quantum crystal is described, in accordance with (3.5)-(3.6), by the Landau-Lifshits equation with a convective term [12]. Notice that expressions (3.11) for the flux densities and the equations of motion (3.5),(3.6) coincide with those obtained earlier for quantum crystals (if we omit the effect of degrees of freedom) [3-4]; for the superfluid phase of ${ }^{3} \mathrm{He}-\mathrm{B}$ (if we neglect elasticity) [5], and for a multisublattice magnet with exchange and magnetoelastic interactions (in the absence of superfluidity) [6].

The thermodynamic relation (3.3) and equations (3.5)-(3.7) indicate that nonequilibrium processes are adiabatic

$$
\dot{\sigma}=\frac{1}{T}\left(\dot{\varepsilon}-\mu \dot{\varrho}-v_{k} \dot{\pi}_{k}-\underline{h}_{\alpha} \underline{\dot{s}}_{\alpha}-\underline{\chi}_{\alpha k} \underline{\dot{\omega}}_{\alpha k}-j_{i}^{s} \dot{p}_{i}-\nu_{i k} \dot{b}_{i k}\right)=-\operatorname{div} \sigma \vec{v}^{n} .
$$

It should be noted that formulae (3.4) for the flux densities can be written in a compact form by introducing the thermodynamic potential density

$$
\omega=Y_{a} \zeta_{a}-\sigma, \quad a=(0, i, \alpha, 4) ; \zeta_{a}=\left(\varepsilon, \pi_{i}, s_{\alpha}, \varrho\right) .
$$

Here $Y_{a}$ are the thermodynamic forces, defined by the relationships

$$
\frac{1}{Y_{0}} \equiv T,-\frac{Y_{4}}{Y_{0}} \equiv \mu,-\frac{Y_{k}}{Y_{0}} \equiv v_{k}^{n},-\frac{Y_{\alpha}}{Y_{0}} \equiv h_{\alpha} .
$$


The second law of thermodynamics in terms of $\omega$ can be written in the form

$$
d \omega=\zeta_{a} d Y_{a}+\frac{\partial \omega}{\partial p_{i}} d p_{i}+\frac{\partial \omega}{\partial b_{i k}} d b_{i k}+\frac{\partial \omega}{\partial \omega_{\alpha k}} d \omega_{\alpha k}+\frac{\partial \omega}{\partial a_{\alpha \beta}} d a_{\alpha \beta} .
$$

It follows from $(3.4),(3.10)$ that

$$
\zeta_{a k}=-\frac{\partial}{\partial Y_{a}} \frac{\omega Y_{k}}{Y_{0}}+\frac{\partial \omega}{\partial \omega_{\alpha k}} \frac{\partial}{\partial Y_{a}} \frac{Y_{\alpha}+Y_{l} \omega_{\alpha l}}{Y_{0}}+\frac{\partial \omega}{\partial p_{k}} \frac{\partial}{\partial Y_{a}} \frac{Y_{4}+Y_{i} p_{i}}{Y_{0}}+\frac{\partial \omega}{\partial b_{j k}} \frac{\partial}{\partial Y_{a}} \frac{b_{j l} Y_{l}}{Y_{0}} .
$$

Let us suppose that the system under investigation possesses the property of Galilean invariance. The thermodynamic potential $\omega$ depends only on the following combinations of thermodynamic forces $Y_{a}[4]$ :

$$
\begin{gathered}
\omega=\omega\left(Y_{0}^{\prime}, Y_{k}^{\prime}, Y_{\alpha}^{\prime}, Y_{4}^{\prime}, b_{i k}, \omega_{\alpha k}\right), \\
Y_{0}^{\prime}=Y_{0}, Y_{k}^{\prime}=Y_{k}+Y_{0} \frac{p_{k}}{m}, Y_{\alpha}^{\prime}=Y_{\alpha}, Y_{4}^{\prime}=Y_{4}+Y_{k} p_{k}+Y_{0} \frac{p^{2}}{2 m} .
\end{gathered}
$$

Noting that

$$
\frac{\partial \omega}{\partial p_{l}}=\frac{Y_{0}}{m} \frac{\partial \omega}{\partial Y_{l}^{\prime}}+\left(Y_{l}+Y_{0} \frac{p_{l}}{m}\right) \frac{\partial \omega}{\partial Y_{4}^{\prime}}, \frac{\partial \omega}{\partial Y_{l}}=\frac{\partial \omega}{\partial Y_{l}^{\prime}}+p_{l} \frac{\partial \omega}{\partial Y_{4}^{\prime}}
$$

we obtain the following expressions for the flux densities:

$$
\begin{gathered}
j_{k}=\frac{1}{m} \frac{\partial \omega}{\partial Y_{k}^{\prime}}+\frac{p_{k}}{m} \frac{\partial \omega}{\partial Y_{4}^{\prime}}, j_{\alpha k}=-\frac{Y_{k}}{Y_{0}} \frac{\partial \omega}{\partial Y_{\alpha}^{\prime}}+\frac{1}{Y_{0}} \frac{\partial \omega}{\partial \omega_{\alpha k}}, \\
t_{i k}=-\frac{\omega}{Y_{0}} \delta_{i k}+\frac{p_{i} p_{k}}{m} \frac{\partial \omega}{\partial Y_{4}^{\prime}}+\frac{p_{i}}{m} \frac{\partial \omega}{\partial Y_{k}^{\prime}}-\frac{Y_{k}}{Y_{0}} \frac{\partial \omega}{\partial Y_{i}^{\prime}}+\frac{\omega_{\alpha i}}{Y_{0}} \frac{\partial \omega}{\partial \omega_{\alpha k}}+\frac{b_{j i}}{Y_{0}} \frac{\partial \omega}{\partial b_{j k}} \\
q_{k}=-\frac{Y_{4}+Y_{l} p_{l}}{m Y_{0}} \pi_{k}-\frac{Y_{k}}{Y_{0}{ }^{2}}\left(\sigma-Y_{l} \frac{\partial \omega}{\partial Y_{l}^{\prime}}\right)-\frac{b_{j l} Y_{l}}{Y_{0}{ }^{2}} \frac{\partial \omega}{\partial b_{j k}}-\frac{Y_{\alpha}+Y_{l} \omega_{\alpha l}}{Y_{0}{ }^{2}} \frac{\partial \omega}{\partial \omega_{\alpha k}} .
\end{gathered}
$$

In this case the second law of thermodynamics can be written in the form

$$
\begin{gathered}
T d \sigma=d \varepsilon+\frac{Y_{k}^{\prime}}{Y_{0}} d \pi_{k}+\frac{Y_{\alpha}^{\prime}}{Y_{0}} d s_{\alpha}+\frac{Y_{4}^{\prime}}{Y_{0}} d \varrho- \\
-\frac{1}{Y_{0}} \frac{\partial \omega}{\partial b_{i k}} d b_{i k}-\frac{1}{Y_{0}} \frac{\partial \omega}{\partial \omega_{\alpha k}} d \omega_{\alpha k}-\frac{1}{Y_{0}} \frac{\partial \omega}{\partial a_{\alpha \beta}} d a_{\alpha \beta} .
\end{gathered}
$$

Let us now consider time independent solutions of equations (3.5)-(3.7), when the quantities $\sigma, \varrho, v_{i}, p_{i}, \underline{s}_{\alpha}, \underline{\omega}_{\alpha k}, b_{i k}$ are independent of coordinates and time (but not the rotational matrix $a$; it will be shown below that this leads to helical magnetic solutions). It can be easily seen that Cartan forms $\underline{\omega}_{\alpha k}$ satisfy the identities

$$
\nabla_{k} \underline{\omega}_{\alpha i}-\nabla_{i} \underline{\omega}_{\alpha k}=\epsilon_{\alpha \beta \gamma} \underline{\omega}_{\beta k} \underline{\omega}_{\gamma i},
$$

which leads to the following identity in the stationary state:

$$
\epsilon_{\alpha \beta \gamma} \underline{\omega}_{\beta k} \underline{\omega}_{\gamma i}=0 .
$$


The stationary conditions for equations (3.5)-(3.7), taking into account expression (3.13), assume the form

$$
\epsilon_{\alpha \beta \gamma} \underline{\omega}_{\beta k} \frac{\partial \varepsilon}{\partial \underline{s}_{\gamma}}=0, \epsilon_{\alpha \beta \gamma}\left(\underline{s}_{\beta} \frac{\partial \varepsilon}{\partial \underline{s}_{\gamma}}+\underline{\omega}_{\beta k} \frac{\partial \varepsilon}{\partial \underline{\omega}_{\gamma k}}\right)=0 .
$$

Expressions (3.13), (3.14) can be used to obtain the structure of Cartan forms in equilibrium

$$
\underline{\omega}_{\alpha k}=n_{\alpha} g_{k}, n_{\alpha}=\frac{\underline{h}_{\alpha}}{|\underline{h}|}, \underline{h}_{\alpha} \equiv \frac{\partial \varepsilon}{\partial \underline{s}_{\alpha}} .
$$

Here the quantity $g_{k}$ has the meaning of the helical vector of the magnetic structure. In order to verify this, we also introduce the right differential Cartan form associated with the time derivative:

$$
\underline{\omega}_{\alpha}=\frac{1}{2} \epsilon_{\alpha \beta \gamma}(\dot{a} \tilde{a})_{\gamma \beta} .
$$

From the equations of motion (3.6) for the matrix $a$ we obtain

$$
\underline{\omega}_{\alpha}=-v_{i} \underline{\omega}_{\alpha i}-\underline{h}_{\alpha},
$$

which leads to the following expression for $\underline{\omega}_{\alpha}$ in equilibrium if we take Eq. (3.15) into account:

$$
\underline{\omega}_{\alpha}=-n_{\alpha}\left(\underline{h}+g_{i} v_{i}\right) .
$$

According to the definitions of the Cartan forms $\underline{\omega}_{\alpha k}, \underline{\omega}_{\alpha}$, the derivatives of the rotational matrix $a$ with respect to coordinates and time can be written in the form

$$
\nabla_{k} a_{\rho \gamma}=-\epsilon_{\rho \mu \alpha} a_{\mu \gamma} \underline{\omega}_{\alpha k}, \dot{a}_{\rho \gamma}=-\epsilon_{\rho \mu \alpha} a_{\mu \gamma} \underline{\omega}_{\alpha} .
$$

Taking the structure (3.15),(3.18) of Cartan's forms in equilibrium into account, we can write the solution to these equations in the form

$$
a(x, t)=a\left(n\left(-g_{i} x_{i}+\left(\underline{h}+g_{i} v_{i}\right) t\right)\right),
$$

which describes the rotation about the direction of the vector $\vec{n}$ through an angle $\theta=-g_{i} x_{i}+\left(\underline{h}+g_{i} v_{i}\right) t$. In equilibrium, the coordinate and time dependence of the spin density $s(x, t)$ is defined by the formula $s(x, t)=$ $\underline{s} a(x, t)$, which proves the validity of the above statement.

\section{Spectrum of elementary excitations}

Obtained equations (3.5)-(3.7) contain all the information needed for calculation the velocities of propagating modes, whose general structure can be easily understood here as coupling of a conserved quantity to an order parameter, leading to propagation of this conserved quantity.

Therefore, in an ordinary crystal, where $j_{i}^{s}=0$, Eqs. for $\pi_{i}(x), b_{i k}(x)$ yield three pairs of elastic waves, which are the propagation of momentum. If we add the superfluid phase $\phi(x)$ (and, respetively, the density of the mass $\varrho(x))$, that corresponds to a quantum crystal then it provides the system with independent persistent mass current $j_{i}^{s}$ and gives rise to the propagation of the mass. If we consider together with above variables the 
density of the spin $s_{\alpha}(x)$ and the matrix of rotation $a_{\alpha \beta}(x)$ we arrive at situation of a quantum spin crystal and obtain additional six branches of spin waves, that relates to the propagation of the spin.

The case of a quantum crystal was studied in Refs. [1-4]. Respective propagating modes are the three elastic waves and the second sound with corresponding velocities [3]

$$
\begin{gathered}
c_{1}^{2}=c_{10}^{2}\left(1+\frac{\varrho_{i j}^{s} \hat{u}_{i} \hat{u}_{j}}{\varrho}\right)+\frac{1}{\varrho}\left(\beta_{i j} \hat{q}_{i} \hat{u}_{j}\right)\left[2\left(\varrho_{i j}^{s} \hat{q}_{i} \hat{u}_{j}\right)+\frac{\varrho_{i j}^{s} \hat{q}_{i} \hat{q}_{j}}{c_{10}^{2}}\left(\beta_{i j} \hat{q}_{i} \hat{u}_{j}\right)\right], \\
c_{2}^{2}=\left(\varrho_{i j}^{s} \hat{q}_{i} \hat{q}_{j}\right)\left[\left(\frac{\partial \mu}{\partial \varrho}\right)_{\sigma, b}-\frac{1}{c_{10}^{2} \varrho}\left(\beta_{i j} \hat{q}_{i} \hat{u}_{j}\right)^{2}\right] .
\end{gathered}
$$

Here $c_{10}$ is the velocity of elastic waves in an ordinary crystal, $\hat{u}$ is the unit polarization vector, $\hat{q}=\vec{q} / q$ the unit wave vector, $\beta_{i j}=-\partial \mu / \partial b_{j i}$ and components of the superfluid tensor $\varrho_{i j}^{s}$ are defined from the relationship

$$
j_{i}^{s}=\varrho_{i j}^{s}\left(v_{j}^{s}-v_{j}^{n}\right), v_{i}^{s} \equiv \frac{p_{i}}{m} .
$$

For finding the spectrum of spin waves in the case of pure spin dynamics, when we neglect weak magnetoelastic interactions, one should use dynamic equations for the variables $\underline{s}_{\alpha}, \underline{\omega}_{\alpha k}$. We consider the equilibrium state characterized by the equilibrium values $\underline{s}_{\alpha}^{0}=0, \underline{h}_{\alpha}=0, g_{k}=0$. Linearizing the motion equations for $\underline{s}_{\alpha}, \underline{\omega}_{\alpha k}$ with account of the relationship [11] $\delta \underline{\omega}_{\alpha k}=\nabla_{k} \delta \phi_{\alpha}-\epsilon_{\alpha \beta \gamma} \underline{\omega}_{\beta k}^{0} \delta \phi_{\gamma}\left(\phi_{\alpha}\right.$ is the rotation angle) we arrive at

$$
\begin{gathered}
\left(-\omega \delta_{\alpha \beta}+G_{\alpha, \beta l} q_{l}\right) i \delta \phi_{\beta}+F_{\alpha \beta} \delta \underline{s}_{\beta}=0, \\
i H_{\alpha k, \beta l} q_{k} q_{l} \delta \phi_{\beta}+\left(-\omega \delta_{\alpha \beta}+G_{\beta, \alpha k} q_{k}\right) \delta \underline{s}_{\beta}=0,
\end{gathered}
$$

where

$$
F_{\alpha \beta}=\frac{\partial^{2} \varepsilon}{\partial \underline{s}_{\alpha} \partial \underline{s}_{\beta}}, G_{\alpha, \beta l}=\frac{\partial^{2} \varepsilon}{\partial \underline{s}_{\alpha} \partial \underline{\omega}_{\beta l}}, H_{\alpha k, \beta l}=\frac{\partial^{2} \varepsilon}{\partial \underline{\omega}_{\alpha k} \partial \underline{\omega}_{\beta l}} .
$$

From the latter we obtain for the spectrum of spin waves

$$
c_{30}=\frac{\omega}{q}=\frac{1}{2}\left\{G_{\alpha, \beta k} \hat{q}_{k}\left(\underline{\hat{s}}_{\alpha} \underline{\hat{s}}_{\beta}+\hat{\phi}_{\alpha} \hat{\phi}_{\beta}\right) \pm\right.
$$

$$
\pm \sqrt{\left.\left(G_{\alpha, \beta k} \hat{q}_{k}\left(\underline{\hat{s}}_{\alpha} \underline{\hat{s}}_{\beta}+\hat{\phi}_{\alpha} \hat{\phi}_{\beta}\right)\right)^{2}+4\left(F_{\alpha \nu} H_{\beta k, \mu l}-G_{\alpha, \beta l} G_{\nu, \mu k}\right) \hat{q}_{k} \hat{q}_{l} \hat{\phi}_{\alpha} \hat{\phi}_{\beta} \underline{\hat{s}}_{\nu} \hat{\underline{s}}_{\mu}\right\}}
$$

$(\underline{\hat{s}}=\underline{\vec{s}} / s$ and $\hat{\phi}=\vec{\phi} / \phi$ are the spin and angle polarization vectors respectively).

Now we consider the quantum spin crystal. The general spectrum of the ten propagating modes is given by solving Eqs. (3.5)-(3.7) simultaneously. These are the three elastic waves, the second sound and the six spin waves. Corresponding analysis is very bulky and comes to some straightforward algebra. By this reason we only outline obtained results in general. After elimination of variations $\delta \varrho$ and $v_{i}^{n}$ (in equilibrium $v_{i}^{n}=0$ ) we come to at the determinant for finding the spectrum with the next structure

$$
\left|\begin{array}{cc}
\text { I } & \text { III } \\
\text { IV } & \text { II }
\end{array}\right|=0 \text {. }
$$


Here I is the block corresponding to the quantum crystal and its structure is written in Ref. [3]; the structure of the second block II is determined by the Eqs. (4.1) and it corresponds to the pure spin dynamics. The blocks III, IV contain the cross derivatives such as $\frac{\partial^{2} \varepsilon}{\partial \underline{s}_{\alpha} \partial \varrho}, \frac{\partial^{2} \varepsilon}{\partial \underline{s}_{\alpha} \partial b}, \frac{\partial^{2} \varepsilon}{\partial \underline{\omega}_{\alpha k} \partial \varrho}$, etc., that corresponds to account of magnetoelastic interactions. $\overline{\mathrm{W}}$ e shall consider these interactions as weak. Thus, propagating modes of quantum crystal and spin waves will be modified by the magnetoelastic interactions. Untill the lowest nonvanishing order in such magnetoelastic terms, the corresponding corrections in expressions for the velocities of propagating modes have the following structure:

\section{1) for elastic waves $\quad \tilde{c}_{1}^{2}=c_{1}^{2}+(\ldots \text { magnetoelast. terms })^{2}$,}

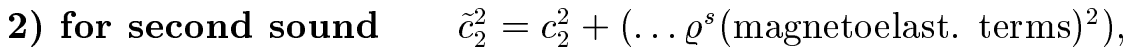

\section{3) for spin waves $\quad \tilde{c}_{3}=c_{3}+(\ldots \text { magnetoelast. terms })^{2}$,}

where we conventionally denote the quadratic terms on derivatives $\frac{\partial^{2} \varepsilon}{\partial \underline{s}_{\alpha} \partial \varrho}$, $\frac{\partial^{2} \varepsilon}{\partial \underline{s}_{\alpha} \partial b}$, etc., as (... magnetoelast. terms) $)^{2}$. We see that the lowest order of corrections to the velocities of elastic and spin waves are proportional to the second order combinations of cross derivatives, corresponding to magnetoelastic interactions. For the second sound such correction contains also as multiplier the component $\varrho_{i j}^{s}$ of the superfluid tensor.

Note in conclusion that because of the correspondence between the conserved quantities and the propagating modes, there can be no more propagating modes than are found here.

\section{Acknowledgments}

The authors are pleased to express their gratitude to S.V. Peletminsky for fruitful discussions of the results and for valuable remarks.

\section{References}

[1] Andreev A.F., Lifshits I.M. Quantum theory of crystal defects. // ZETP, 1969, vol. 56, No 6, p. 2057-2068 (in Russian).

[2] Saslow W.M. Microscopic and hydrodynamic theory of superfluidity in periodic solids. // Phys. Rev. B, 1977, vol. 15, p. 173-186.

[3] Liu M. Two possible types of superfluidity in crystals. // Phys. Rev. B, 1978, vol. 18 , No 3 , p. 1165-1176.

[4] Lavrinenko N.M., Peletminsky S.V. Thermodynamics and equations of motion for quantum crystals. // Teor. Mat. Fiz., 1986, vol. 66, No 2, p. 314-324 (in Russian).

[5] Maki K. General gauge invariance and spin waves in the B-phase of superfluid ${ }^{3}$ He. // Phys. Rev. B, 1975, vol. 11, p. 4264-4271.

[6] Virchenko Yu.P., Peletminsky S.V. Poisson brackets and differential conservation laws in the theory of magnetoelastic media. In: Problems of physical kinetics and solid-state physics. Kiev, 1990 (in Russian).

[7] Dzyaloshinsky I.E., Volovick G.E. Poisson brackets in condensed matter physics. // Ann. Phys., 1980, vol. 125, No 1, p. 67-97. 
[8] Kats E.I., Lebedev V.V. Fluctuational effects in the dynamics of liquid crystals. Springer, 1993.

[9] Kovalevsky M.Yu., Peletminsky S.V., Shishkin A.L. Hamiltonian formalism for magnetic systems with spontaneously broken symmetry. // Ukr. Fiz. Zh., 1991, vol. 36, No 2, p. 245-260 (in Russian).

[10] Isayev A.A., Kovalevsky M.Yu., Peletminsky S.V. Hamiltonian dynamics of the condensed media with spontaneously broken symmetry. // Preprint IC/94/329, Miramare-Trieste, 1994.

[11] Kovalevsky M.Yu., Rozhkov A.A. On the theory of disorded magnetics. // Physica A, 1995, vol. 216, p. 169-184.

[12] Akhiezer A.I., Bar'yakhtar V.G., Peletminsky S.V. Spin Waves. Amsterdam, North Holland, 1960.

\title{
ДО ГІДРОДИНАМІЧНОЇ ТЕОРІЇ КВАНТОВИХ СПІНОВИХ КРИСТАЛІВ
}

\author{
А.А.Ісаєв, М.Ю.Ковалевський
}

На основі гамільтонового підходу отримуються гідродинамічні рівняння для квантового спінового кристалу. Формулюється основна термодинамічна тотожність. Вирази для густин потоків додаткових інтегралів руху знайдено через термодинамічні потенціали густини як для галілеєво інваріантних систем, так і для галілеєво неінваріантних систем. Отримано стаціонарний розв'язок, що відповідає спіральному магнітному впорядкуванню. Досліджується спектр мод, що поширюються. 